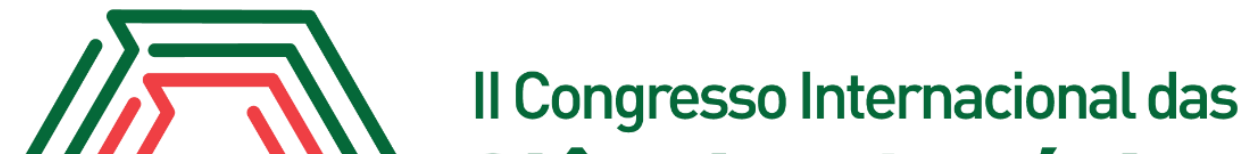 Ciências Agrárias COINTER - PDVAgro 2017
}

\section{RESPOSTA DA CULTURA CRAMBE A FERTILIZAÇÃO NITROGENADA}

Apresentação: Comunicação Oral

José Travassos Dos Santos Júnior ${ }^{1}$; Harielly Tyanne Gabriel Targino ${ }^{2}$; Eshiley Eduarda de Oliveira Torres $^{3} ;$ Rogerio Dantas de Lacerda ${ }^{4}$;

\section{Resumo}

Este trabalho estudou o manejo da cultura do Crambe (Crambe abyssinica) em condições de campo, visando à otimização da adubação nitrogenada, sobre seu crescimento/desenvolvimento. $\mathrm{O}$ experimento foi desenvolvido na área experimental do Instituto Federal de Educação, Ciência e Tecnologia do Rio Grande do Norte- IFRN, Apodi- RN. O ensaio foi conduzido com o cultivar de Crambe FMS Brilhante submetido a quatro diferentes doses de nitrogênio $(25 ; 50 ; 75$ e 100 kg ha-1 de Nitrogênio). Os dados foram analisados estatisticamente, através do qual realizou-se a analise de variância aplicando-se o teste F. Verificou-se que os fatores de crescimento da cultura como altura da planta, área foliar, número de ramos e número de cápsulas foram influenciadas pelos níveis de nitrogênio aplicado ao solo.

Palavras-Chave: biodiesel, desenvolvimento sustentável, manejo de água e solo.

\section{Introdução}

O Crambe (Crambe abyssinica Hortsh Ex. R. T), planta da família Brassicaceae, de origem mediterrânea, caracteriza-se por ser um vegetal arbustivo, de ciclo curto, uma vez que floresce em 35 dias e pode ser colhido em 90 dias, em virtude de se adaptar a condições climáticas diferenciadas, desde geadas típicas do sul do País até climas quentes e secos do cerrado brasileiro, em razão do baixo custo de cultivo e porque a maturidade uniforme possibilita a realização de colheita mecanizada (Falasca et al., 2010).

\footnotetext{
${ }^{1}$ Técnico integrado em Agricultura, Instituto Federal de Educação Ciência e Tecnologia do Rio Grande do Norte IFRN, E-mail: travassosjunior96@gmail.com

${ }^{2}$ Técnico integrado em Agricultura, Instituto Federal de Educação Ciência e Tecnologia do Rio Grande do Norte IFRN, E-mail: hariellytyanne15@gmail.com

${ }^{3}$ Técnico integrado em Agricultura, Instituto Federal de Educação Ciência e Tecnologia do Rio Grande do Norte IFRN, E-mail: eshiley73@gmail.com

4 Doutor, Instituto Federal de Educação Ciência e Tecnologia do Rio Grande do Norte IFRN, E-mail: rogerio dl@yahoo.com.br
} 
O Crambe (Crambe abyssinica Hochst) é uma espécie vegetal da família das crucíferas. Tem-se como seu local de origem a região do Mediterrâneo, mas a espécie tem demonstrado boa adaptação à diferentes condições climáticas (SOUZA et al., 2009).

No Brasil, é notória a adaptabilidade ao clima, rusticidade, precocidade, tolerância ao déficit hídrico e, a sua principal característica, ter ciclo de produção reduzido, cerca de 90 dias. Logo, o Crambe apresenta-se como uma excelente alternativa para a rotação de culturas com grande potencial de expansão como alternativa de safrinha em boa parte do cerrado brasileiro, não competindo com as culturas principais e as alimentares, e tendo seu cultivo totalmente mecanizado com equipamentos utilizados em outros cultivos, como na soja, utilizando as mesmas estruturas (semeadoras, colhedoras, armazéns) (ROSCOE \& DELMONTES, 2008).

O óleo refinado tem potencial para uso farmacêutico e também na alimentação de pescado. O subproduto da extração do óleo, a torta e/ou farelo, podem ser utilizados como suplementos proteicos em alimentação de ruminantes. $\mathrm{O}$ farelo apresenta excelente qualidade nutricional, com índices de até $45 \%$ de proteína bruta, com digestibilidade ruminal da matéria orgânica semelhante ao farelo de soja (CARLSON et al., 1996).

O óleo de Crambe não pode ser utilizado para o consumo humano, devido à presença de altos teores de ácido erúcico, um ácido graxo monoinsaturados de cadeia longa. Esse ácido provoca lesões no coração quando presente no organismo humano. Assim, este o óleo não concorre com óleos destinados ao setor alimentício (ABROL et al., 1997). O presente trabalho verificou o comportamento da cultura do Crambe quanto submetida a diferentes níveis de adubação a base de nitrogênio.

\section{Fundamentação Teórica}

As sementes apresentam maior percentagem de germinação e vigor por ocasião do ponto de maturidade fisiológica. A partir deste instante, ocorrem, inevitavelmente, mudanças fisiológicas e bioquímicas graduais, que ocasionam a deterioração e a perda gradativa do vigor (Carvalho \& Nakagawa 2000).

Além disso, o crambe se destaca por boa adaptação, rusticidade, precocidade e bom potencial produtivo (Plein et al., 2010). O seu teor de óleo é de aproximadamente 40\% (Bondioli et al, 1998), sendo constituído por mais de 55\% de ácido erúcico (Bondioli et al, 1998; Yaniv et $a l, 1998)$, que é um ácido graxo de cadeia longa com alto valor industrial. Sua boa capacidade de secagem e habilidade em polimerizar-se permite ser usado como um óleo para a formação de pastas 
em pintura (binder), como lubrificante industrial, como inibidor da corrosão e como um ingrediente para a fabricação de borracha sintética. Ele também pode ser utilizado para produzir filmes plásticos, plastificantes, nylon, adesivos e para isolamento elétrico. Também pode ser utilizado na fabricação de produtos químicos intermediários que, posteriormente, serão utilizados como cosméticos, produtos de higiene pessoal, entre outros (Lazzeri et al., 1997).

Para Malavolta (1980) a planta necessita de elementos minerais essenciais, como o nitrogênio, fósforo e potássio (NPK) e quando um destes não está disponível, a planta não completa seu ciclo de vida. Em oleaginosas, o nitrogênio (N) influência no metabolismo de síntese de compostos de reservas das sementes, determinando os teores de proteínas nos grãos e a produção de óleo (Castro et al, 1999). O fósforo (P), aplicado em quantidades adequadas, estimula o desenvolvimento radicular, propicia maior vigor, acelera a maturação físiológica, incita o florescimento, formação das sementes, aumenta a resistência ao frio e produtividade (Malavolta, 1989). Já o potássio (K) participa da maioria dos processos biológicos em uma planta e quando não disponibilizado na dose mínima pode reduzir desenvolvimento da cultura e consequentemente, a produtividade (Malavolta, 1997; Castro e Oliveira, 2005).

As pesquisas com o Crambe e a sua produção comercial se intensificaram a partir dos anos 80, após sua introdução nos Estados Unidos da América, no Reino Unido e em alguns países da Europa, como a Itália, França e Portugal. Contudo, as áreas plantadas nesses países não aumentaram expressivamente, porque essa planta compete por área com as principais culturas de safra, como o milho, a soja e o trigo, sendo que nessas regiões não é possível cultivar o Crambe em safrinhas (período de entressafra compreendido entre as culturas principais e iniciada após a cultura de verão). Dessa forma, o crambe vem se difundindo para outros países, como a Austrália, a África do Sul, o Paraguai e o Brasil (PITOL et al., 2010b).

No Brasil, é notória a adaptabilidade ao clima, rusticidade, precocidade, tolerância ao déficit hídrico e, a sua principal característica, ter ciclo de produção reduzido, cerca de 90 dias. Logo, o Crambe apresenta-se como uma excelente alternativa para a rotação de culturas com grande potencial de expansão como alternativa de safrinha em boa parte do cerrado brasileiro, não competindo com as culturas principais e as alimentares, e tendo seu cultivo totalmente mecanizado com equipamentos utilizados em outros cultivos, como na soja, utilizando as mesmas estruturas (semeadoras, colhedoras, armazéns) (ROSCOE \& DELMONTES, 2008).

\section{Metodologia}


O experimento foi desenvolvido na área experimental do Instituto Federal de Educação, Ciência e Tecnologia do Rio Grande do Norte- IFRN. Apodi - RN, cuja altitude média é de 116 m. Instalação e condução do experimento

A área foi irrigada através de um sistema pressurizado localizado por micro aspersão. As necessidades hídricas diárias da cultura foram determinadas através do balanço de água no solo obtidas a partir dos dados de ETo, determina pela equação Penman-Montheith, com os dados coletados na estação meteorológica automática da Empresa Estadual de Pesquisa Agropecuária EMPARN no município de Apodi - RN, obtido através de medições diárias.

O cultivo foi conduzido utilizando o cultivar de Crambe FMS Brilhante, em condições de campo. O solo foi arado com o auxilio de enxada rotativa, posteriormente após a germinação foram realizadas as adubações de acordo com as recomendações do sistema de produção para o Crambe da FMS (doses de $\mathrm{N}$ de 25, 50, 75 e $100 \mathrm{~kg} \mathrm{ha}^{-1}$ de $\mathrm{N}$, mantendo-se fixas as doses de $\mathrm{P}\left(40 \mathrm{mg} \mathrm{dm}^{3} \mathrm{de}\right.$ $\mathrm{P}_{2} \mathrm{O}_{5}$ ) e $\mathrm{K}\left(40 \mathrm{mg} \mathrm{dm}{ }^{3}\right.$ de $\mathrm{K}_{2} \mathrm{O}$ ). Em seguida o solo foi irrigado elevando o conteúdo de água do solo ate a capacidade de campo de forma a promover a germinação das sementes. O plantio foi realizado a profundidade da semeadura de $1,0 \mathrm{~cm}$.

Para irrigação das plantas foi utilizada água proveniente de poço tubular da área, sendo o sistema de irrigação composto por um conjunto eletrobomba, que conduzira a água pressurizada ate a área experimental onde a distribuição às parcelas será controlada por registros e fornecida às plantas através de micro aspersores. As irrigações foram realizadas de acordo com os tratamentos pré-estabelecidos para o manejo da água, sendo o volume calculado em função do conteúdo de água presente no solo através de medições feitas do balanço de água no solo obtido por medições diárias, através do balanço de água no solo obtido por medições diárias da Evapotranspiração de referencia - ETo, através do método de Penman-Montheith.

Aos 30 dias apos a semeadura (DAS) será realizada a primeira coleta de dados para determinada o do desempenho de crescimento e desenvolvimento das plantas de Crambe, assim procedendo-se aos 60 dias. Ao final ciclo por volta dos 90 dias procedeu-se a coleta final da produção.

O experimento foi desenvolvido em condições de campo em um delineamento experimental de blocos ao acaso, em três blocos e 4 tratamentos, sendo 4 doses nitrogenadas. Constituindo 12 parcelas iguais de $40 \mathrm{~m}^{2}$, as quais ocuparão uma área total de $480 \mathrm{~m} 2$.

As lâminas de irrigação aplicadas ao cultivar de Crambe neste ensaio foram baseadas no diferentes níveis de reposição de água de irrigação em função da Evapotranspiração da cultura ETc, utilizando-se a Evapotranspiração de referencia - ETo, através do método de Penman-Montheith. 
O cálculo da lâmina de água a aplicada as plantas foi baseada na equação a seguir (ALLEN et al., 1998):

$$
\mathrm{ETc}=\text { Kc.ETo }
$$

Onde:

$\mathrm{ETc}=$ evapotranspiração da cultura (mmdia-1);

Kc $=$ coeficiente de cultivo de acordo com estádio de desenvolvimento da cultura (adimensional);

ETo $=$ evapotranspiração potencial Penman Montheith $\left(\mathrm{mmdia}^{-1}\right)$.

De acordo com a FAO (2002), foram utilizado Kc máximo para cada estádio de desenvolvimento: estágio inicial - 20 a 25 dias - $(\mathrm{Kc}=0,4)$; $)$ estágio vegetativo - 35 a 40 dias - (Kc $=0,8)$; florescimento -40 a 50 dias - $(\mathrm{Kc}=1,2)$; enchimento de grãos -25 a 30 dias $-(\mathrm{Kc}=0,8)$ e maturação fisiológica $(\mathrm{Kc}=0,4)$.

Cada parcela experimental foi constituída por uma área de 10,00 m2, onde foram semeadas 30 sementes por metro linear, espaçadas em 0,7 metros entre fileiras. Os dados foram coletados e analisados estatisticamente do qual foi feita a analise de variância (ANAVA) aplicando-se o teste F e analise regressão para o fator quantitativo (FERREIRA, 2000).

\section{Resultados e Discussões}

Na comparação das médias dos tratamentos para os níveis de adubação nitrogenada, referente à altura da planta aos 30 e 60 DAS podem ser observadas na Tabela 1. As plantas apresentaram altura máxima de 17,00 e 87,90 cm aos 30 e 60 DAS respectivamente, com o nível de $75 \mathrm{~kg}$ ha-1 de N.

Tabela 1. Médias da variável altura da planta do cultivar de Crambe FMS Brilhante, quando submetidos a diferentes níveis de adubação nitrogenada (N) aos 30 e 60 DAS. IFRN, 2015.

\begin{tabular}{ccc}
\hline \multirow{2}{*}{$\left(\mathrm{kg} \mathrm{ha}^{-1}\right)$} & \multicolumn{2}{c}{ Médias da altura das plantas $(\mathrm{cm})$} \\
\cline { 2 - 3 } & 30 DAS & 60 DAS \\
\hline 25 & 12,00 & 80,22 \\
50 & 14,02 & 83,70 \\
75 & 17,00 & 87,90 \\
100 & 14,80 & 77,90 \\
\hline
\end{tabular}


Chaves e Ledur (2014) avaliando em campo experimental no Estado do Paraná, verificou que a altura das plantas foi influenciada pela aplicação de fósforo e pela interação de $\mathrm{N}$ com P, já Maekawa Junior et al. (2010) não encontraram influência da adubação sobre a altura das plantas de Crambe.

As médias dos tratamentos os níveis de adubação nitrogenada, referente ao número de ramos aos 30 e 60 DAS podem se observadas na Tabela 2. Houve diferenças significativas nas médias referentes aos números de ramos aos 30 e 60 DAS.

Tabela 2. Médias da variável número de ramos do cultivar de Crambe FMS Brilhante, quando submetidos a diferentes níveis de adubação nitrogenada (N) aos 30 e 60 DAS. IFRN, 2015.

\begin{tabular}{ccc}
\hline \multirow{2}{*}{$\mathrm{N}\left(\mathrm{kg} \mathrm{ha}^{-1}\right)$} & \multicolumn{2}{c}{ Médias do número de ramos } \\
\cline { 2 - 3 } & 30 DAS & 60 DAS \\
\hline 25 & 7,50 & 12,10 \\
50 & 9,50 & 12,87 \\
75 & 10,81 & 13,43 \\
100 & 9,88 & 14,22 \\
\hline
\end{tabular}

Chaves e Ledur (2014) encontraram em pesquisa realizada no Estado do Paraná que o número de ramificações foi significativamente influenciado, ao nível de $1 \%$, pela aplicação de nitrogênio e de fósforo na cultura do Crambe.

As médias dos tratamentos os níveis de adubação nitrogenada, referente ao diâmetro do caule aos 30 e 60 DAS podem ser observadas na Tabela 3.

Tabela 3. Médias da variável área foliar do cultivar de Crambe FMS Brilhante, quando submetidos a diferentes níveis de adubação nitrogenada (N) aos 30 e 60 DAS. IFRN, 2015.

\begin{tabular}{ccc}
\hline \multirow{2}{*}{$\left(\mathrm{kg} \mathrm{ha}^{-1}\right)$} & \multicolumn{2}{c}{ Médias da área foliar $\left(\mathrm{cm}^{2}\right)$} \\
\cline { 2 - 3 } & 30 DAS & 60 DAS \\
\hline 25 & 445,00 & 192,00 \\
50 & 389,00 & 165,00 \\
75 & 340,00 & 158,00 \\
100 & 402,00 & 210,00 \\
\hline
\end{tabular}

As médias diferiram estatisticamente na variável área foliar, quando submetidas a diferentes níveis de N, obtendo-se uma maior área aos 30 DAS com 445,00 cm² e $25 \mathrm{~kg} \mathrm{ha}^{-1}$ de N e 192,00 e 
$192,33 \mathrm{~cm}^{2}$ com 25 e $100 \mathrm{~kg} \mathrm{ha}^{-1}$ de $\mathrm{N}$ respectivamente.

\section{Conclusões}

A adubação nitrogenada com níveis ascendentes proporcionou aumento nas variáveis de crescimento e/ou desenvolvimento área foliar e numero de ramos. Para a altura da planta houve decréscimos a partir do nível de $75 \mathrm{~kg} \mathrm{ha}^{-1}$ de $\mathrm{N}$.

\section{Referências}

BONDIOLI, P.; FOLEGATTI, L.; LAZZERI, L.; PALMIERI, S.; Native Crambe abyssinica oil and its derivatives as renewable lubricants: an approach to improve its quality by chemical and biotechnological processes; Industrial Crops and Products, 1998, 7, p 231-238.

CARVALHO, N. M.; NAKAGAWA, J. Sementes: ciência, tecnologia e produção. 4. ed. Jaboticabal: Funep, 2000.

DESAI, B. B.; KOTECHA, P.M.; SALUNKHE, D. K. Seeds handbook: biology, production processing and storage. New York: Marcel Dekker, 1997. 627 p.

FALASCA, S. L.; FLORES, N.; LAMAS, M. C.; CARBALLO, S. M.; ANSCHAU, A. Crambe abyssinica: An almost unknown crop with a promissory future to produce biodiesel in Argentina. International Journal of Hydrogen Energy, v.35, p.5808-5812, 2010.

JASPER, S. P.; BIAGGIONI, M. A. M.; SILVA, P. R. A. Comparação do custo de produção do crambe (Crambe abyssinica Hochst) com outras culturas oleaginosas em sistema de plantio direto. Revista Energia na Agricultura, Botucatu, vol. 25, n.4, 2010, p.141-153.

LAZZERI, L.; DE MATTEI, F.; BUCELLI, F.; PALMIERI, S.; Crambe oil - a potential new hydraulic oil and quenchant; Industrial Lubrication and Tribology, 1997, 49, 2, p 71-77.

OLIVEIRA, R. C. e outros. Agro industrialização do Crambe. 2 ed. Cascavel: ASSOESTE, 2015. OLIVEIRA, R. C. e outros. Cultura do Crambe. 1 ed. Cascavel: ASSOESTE, 2013.

OPLINGER, E.S. et al. Crambe, alternative field crops manual. University of Wisconsin and University of Minnesota St. Paul, MN 55108. July, 1991. Disponível em: <http://www.hort.purdue.edu/newcrop/AFCM/crambe.html> Acesso em: 02 de julho de 2017. 
PITOL, C.; Cultura do Crambe. In: Tecnologia e Produção: Milho Safrinha e Culturas de Inverno 2008. 1. ed. Maracajú: Fundação MS, 2008. v.1. c.11, p.85-88. Disponível em: Acesso em: 16 de agosto 2017 .

PLEIN, G. S.; FAVARO, S. P.; DE SOUZA, A. D. V.; DE SOUZA, C. F. T.; CICONINI, G.; DOS SANTOS, G. P.; MIYAHIRA, M. A. M.; ROESCOE, R.; Caracterização da Fração Lipídica em Sementes de Crambe Armazenadas com e sem Casca; IV Congresso Brasileiro de Mamona e I Simpósio Internacional de Oleaginosas Energéticas, João Pessoa, PB, 2010. Disponível em: http://www.cbmamona.com.br/pdfs/OLE-17.pdf Acessada em agosto de 2017.

SILVA, P. R.; MONTANHER, A. F.; ADÃO, D. C.; ZAGONEL, G. F.; et al. Caracterização físico-química de óleo e biodiesel metílico de crambe. $3^{\circ}$ Congresso da Rede Brasileira de Tecnologia de Biodiesel - RBTB. Brasília - DF. 2009.

YANIV, Z.; SHABELSKY, E.; SCHAFFERMAN, D.; GRANOT, I.; KIPNIS, T.; Oil and fatty acid changes in Sinapis and Crambe seeds during germination and early development; Industrial Crops and Products, 1998, 9, p 1-8

ZOBIOLE, L.H.S. et al. Marcha de absorção de macronutrientes na cultura do girassol. Revista Brasileira de Ciência do Solo, v.34, p.425-433, 2010. Disponível em: <http://www.scielo.br/scielo.php?script=sci_abstract\&pid=S0100-

$\underline{06832010000200016 \& \operatorname{lng}=\mathrm{pt} \& n r m=i s o \& t \operatorname{lng}=\mathrm{pt}}$ 